\title{
Psychophysical maps for subadditive dissimilarity ratings
}

\author{
PETER H. SCHÖNEMANN and ALEJANDRO LAZARTE \\ Purdue University, West Lafayette, Indiana
}

\begin{abstract}
Earlier findings on pairwise dissimilarity ratings of "flat" rectangles (Schönemann, Dorcey, \& Kienapple, 1985) were replicated with a larger stimulus set, which included squares and "tall" rectangles. The data were again strongly subadditive and indicated systematic individual differences in judgment strategies. A metric for bounded response scales (MBR) with slightly reduced upper bound gave the best fit among several negatively accelerated response functions that might account for the subadditivity. Simple four-parameter psychophysical maps of the general form $d=9 \tanh \left(\Sigma_{k} a_{k} x_{k}\right), k=1,4$ (where the $x_{k}$ are simple functions of physical height and width, and the $a_{k}$ parameters are of the order of 2 ) reproduced the 120 average ratings within each strategy group with squared etas in the high 90 s. These results suggest that subjects use simple composition rules for rating dissimilarities of rectangles. The judgments can be explained without appeal to any global internal spatial representation of the stimuli solely in terms of the constraints of the pairwise rating task. (In an Appendix, the MBR is generalized to $m>2$ dimensions.)
\end{abstract}

The purpose of this study was twofold: (1) to replicate the major findings of an earlier report (Schönemann, Dorcey, \& Kienapple, 1985) on dissimilarity ratings of rectangles, and (2) to extend them in such a way that the observed dissimilarity ratings could be explained as explicit functions of the metric parameters defining the stimuli.

\section{Background}

Our interest in this problem area was stimulated by a paper by Krantz and Tversky (1975), who had their subjects rate the pairwise dissimilarities for 17 "flat" rectangles. When a rhomboid stimulus design in a logheight/log-width $(\log \mathrm{H} / \log \mathrm{W})$ coordinate system was mapped into a two-dimensional (2-D) subjective space recovered with a multidimensional scaling (MDS) program, the solution was no longer rhomboid, but trapezoidal, in appearance. Krantz and Tversky concluded that (1) area and shape, rather than height and width, determined the subjects' responses, and (2) subjective area and shape interacted, and shape differences increased with area. Similar results had been reported earlier by Sixtl and Wender (1964) and Wender $(1969,1971)$.

This interaction effect proved elusive in subsequent attempts to replicate it. Working with two different designs, a completely crossed height/width (HW) design and a completely crossed area/shape (AS) design, Borg and Leutner (1983) found no such interaction effect in the HW group, and only a weak interaction in the AS group.

We would like to thank Associate Editor M. Teghtsoonian and two anonymous reviewers for numerous suggestions for improving this manuscript, and Tim Dorcey for help with computer analyses at the early stages of this research project. Requests for reprints should be addressed to Peter H. Schónemann at the Department of Psychological Sciences, Purdue University, West Lafayette, IN 47907.
Wiener-Ehrlich (1978), on the other hand, found an interaction for height and width, but not for area and shape. Schönemann and Borg (1981) produced an AS interaction from Krantz and Tversky's (1975) stimulus design alone, that is, without any data whatsoever, by simply plotting the stimuli in an HW (rather than a $\log \mathrm{H} / \log W$ ) coordinate system. This suggests that the presumed AS interaction may have been an artifact caused by the logarithmic prescaling of the design coordinates.

Subadditivity. On collecting new data that might shed further light on these problems (Schönemann, Dorcey, \& Kienapple, 1985), we noticed, first of all, consistent individual differences between the rating strategies employed by the subjects: Some subjects tended to use height and width in their dissimilarity ratings, others tended to use predominantly shape in preference over area, and still others tended to emphasize area over shape. Thus, previous investigators, who averaged over all their subjects before analyzing the data, may have been dealing with strategy mixtures representing none of the individual subjects.

Second, we noticed an entirely different empirical regularity, which apparently had also been overlooked previously. The dissimilarity ratings actually observed for collinear triples were consistently subadditive, both along the sides and along the diagonals of the design configuration. Concretely, if three stimuli, $A, B$, and $C$, varied only in width, then the sum of the dissimilarity ratings from $A$ to $B$ and from $B$ to $C$ always exceeded the dissimilarity rating from $A$ to $C$, with analogous results for triples that varied only in height and for those located along the two main diagonals of an $\mathrm{HW}$ design. The extent of this subadditivity effect can be assessed with the simple function 


$$
f:=a+b-c,
$$

in which the symbol " : =" stands for " is defined as," $a$ and $b$ are the dissimilarity ratings between two neighboring stimuli, and $c$ is the dissimilarity assigned to the outer pair [i.e., $a:=d(A, B), b:=d(B, C), c:=d(A, C)$ in the above example]. The distributions of this function along height, width, area, and shape have consistently positive means regardless of the direction of the comparison (see, e.g. , Schönemann \& Kienapple, 1984, p. 503, $\mathrm{Abb}, 5)$. If the observed ratings are rescaled into the halfopen $[0,1$ ) interval (by dividing out the upper bound of the response scale: "relative distances"), then the positive bias is of the order of .2. Since then, we have uncovered the same subadditivity effect in several other data sets (e.g., Borg \& Leutner, 1983; Noma \& Johnson, 1977; and our own). This pervasive subadditivity conflicts with the widely held belief that subjects employ some version of the Minkowski power metrics to arrive at their ratings. Because all Minkowski metrics are intradimensionally additive, the expected value of $f$ along the dimensions the subjects actually used would be 0 , not .2 .

Metrics for bounded response scales (MBR). To explain the subadditivity in category ratings of dissimilarity, Schönemann $(1982,1983)$ suggested that it might be a ceiling effect caused by the upper bound of the rating scale $(9$, in our case). More specifically, he advanced the "MBR hypothesis," which states that subjects confronted with a dissimilarity rating task use only simple concatenation rules, such as geometric addition, to combine the projections, $a$ and $b$, of the stimulus interval on the underlying subjective dimensions. Although, superficially, this hypothesis seems to favor the so-called city-block metric,

$$
d:=a+b
$$

as a model for the observed dissimilarity ratings, some adjustment is needed to ensure that $d$ does not exceed the experimenter-imposed upper bound of the rating scale, when either $a$ or $b$, or both, are close to it. This can be accomplished by revising Equation 2 into a subadditive MBR:

$$
d:=(a+b) /(1+a b), \quad 0 \leq a, b<1 .
$$

Formally, Equation 3 is equivalent to the addition rule for hyperbolic tangents. Some formal properties of this simple concatenation in two dimensions and its 1-D analogue were discussed in Schönemann (1983). See the Appendix for the $m$-dimensional generalization. In particular, Equation 3 was indeed shown to be a distance function and to emulate the city-block metric (Equation 2) at the lower end of the $[0,1)$ interval, the so-called sup-metric $[d:=\sup (a, b)]$ at the upper end, and the Euclidean distance $\left[d:=\left(a^{2}+b^{2}\right)^{1 / 2}\right]$ in the middle range. In terms of hyperbolic tangents, Equation 3 can be rewritten as

$$
\begin{gathered}
d=\tanh (u+v), u=\tanh (a), v=\tanh (b), \\
0 \leq d<1
\end{gathered}
$$

where $u$ and $v$ are hyperbolic angles. Hence, the inverse transform of the observed relative distances, $d$,

$$
\tanh ^{-1}(d)=u+v, \quad 0 \leq d<1,
$$

leads back to the city-block metric (Equation 3). The MBR is therefore relatively easy to apply. The inverse hyperbolic tangent function is known to psychologists as Fisher's Z-transformation,

$$
\tanh ^{-1}(x)=.5 \ln [(1+x) /(1-x)] .
$$

Programs for fitting the city-block metric are widely available. ${ }^{1}$ Thus, the MBR hypothesis predicts that the fit of the city-block metric to the observed dissimilarities should markedly improve if the dissimilarities are preprocessed with the inverse hyperbolic tangent transformation. If the model (Equation 3 ) fits, the observed dissimilarity ratings $d$ define the $a$ and $b$ on an absolute scale.

Summary of the main empirical results of our earlier studies. To provide a frame of reference for evaluating our new results, we briefly summarize the major results of our previous rectangle studies (Schönemann, Dorcey, \& Kienapple, 1985; Schönemann \& Kienapple, 1984), which the present study is meant to replicate and extend.

1. As already noted, we found systematic strategy differences among subjects. The largest group used height and width, a smaller group used predominantly shape, and a still smaller group used mainly area. These strategy groups were defined operationally in terms of the contrast of the two largest diagonal ratings.

2 . Within subjects, the data were consistently subadditive along height and width and along area and shape.

3. To account for the subadditivity, eight different subadditive concatenations were fitted to the data. Both in terms of bias (sign and magnitude of the residuals) and in terms of the sums of squared residuals, the city-block metric fit poorest, whereas an MBR with a slightly reduced upper bound fit best. An average power rule placed third. An averaging rule was too subadditive.

4. At the subgroup level, the simpler MBR with the upper bound of the response scale did best. The MDS solutions confirmed our prediction that preprocessing the data with the inverse hyperbolic tangent transformation markedly improved the fit. It also tended to straighten out previously curved lines, essentially reproducing the square stimulus design for the largest group, which used height and width equally.

\section{Stimulus Design of the Present Study}

Because the present study was intended to be a partial replication of the previously reported results, our procedure was similar, except for one important detail: Following Krantz and Tversky (1975), our first study (Schönemann et al., 1985) employed mainly flat rectangles, that is, stimuli for which width exceeded height. As already mentioned, this design gave rise to three basic strategy groups. One may ask, then, what happens to the AS group if the design is enlarged to include both flat and tall rectangles? Three possible outcomes are suggested: 


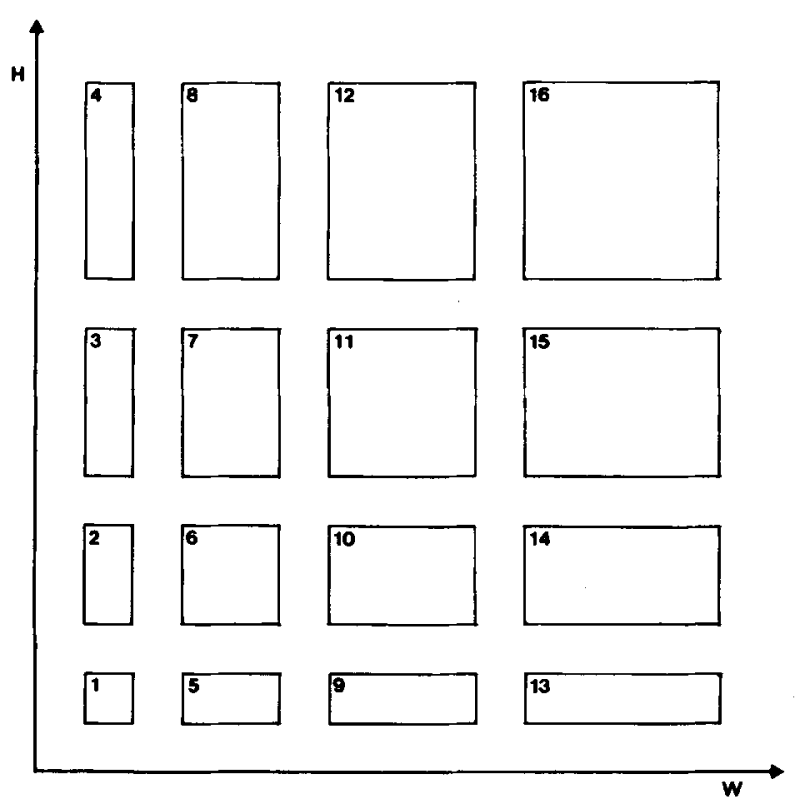

Figure 1. Stimulus design. Stimulus design and numerical labels of rectangles used in text, tables, and figures. The main diagonal from Stimulus 4 to Stimulus 13 is the "shape diagonal" of the design: the minor diagonal from Stimulus 1 to Stimulus 16 is the "area diagonal" of the design. Stimulus 1, the smallest square, measured $2 \times 2 \mathrm{~cm}$ on the screen. Both height and width increased in equal steps by $2 \mathrm{~cm}$.

(1) Subjects in this group continue to use area and shape (shape may relate to either an HW ratio or a difference), so that they distinguish between tall and flat rectangles of the same eccentricity (departure from squareness); (2) they disregard the $90^{\circ}$ rotation that distinguishes flat from tall rectangles, and thus work with a smaller quotient set of 10 , rather than 16 , discriminable stimuli; or (3) this group splits into two subgroups, with some subjects adopting the former and others the latter strategy.

Because we interpreted dissimilarity rating tasks that leave the criterion unspecified ("dissimilar in what way?') to be concept identification tasks that invite individual strategy differences (Schönemann et al., 1985, p. 16), it became important to know whether strategy differences depended on the stimulus design. We therefore extended our new design to include both tall and square rectangles. The stimulus design and the labels we used to identify the rectangles are shown in Figure 1.

The inclusion of squares further removed any ambiguity about the underlying judgment dimensions that plagued us in the study (Schönemann et al., 1985) limited to flat rectangles. For squares, only one possible dimensionsize-however defined, can affect the discrimination. It thus becomes easier to track down the psychophysical map between stimulus dimension and dissimilarity ratings: Are subjects really using area, a quadratic function of linear extent (e.g., W), as an indicator of subjective size, or do they use a linear function of physical width?

\section{METHOD}

\section{Subjects}

The subjects were 43 unpaid volunteers of both sexes, usually of college age. No records were kept of their identities, sexes, or ages.

\section{Apparatus and Stimuli}

Sixteen solid gray rectangles against a dark background on the screen of a TRS-80 PC were presented in pairs to the subjects. The stimulus design was $4 \times 4$, completely crossed in a HW coordinate system. The values for both width and height were $2,4,6$, and $8 \mathrm{~cm}$. In each presentation, both rectangles appeared side by side. The presentation sequence was random, with both pairs, $(x, y)$ and $(y, x)$, presented once, so that there were exactly two replications per subject. Identical pairs $(x, x)$ were omitted.

\section{Procedure}

The computer displayed instructions for the subjects to rate the dissimilarity of each pair on a scale from 0 (identical) to 9 ( $\max$ imally different). This rating scale was also displayed on top of the monitor. To familiarize the subjects with the procedure and the stimulus range, the experimenter first exposed a sequence of roughly 10 pairings in short succession, and then requested the subject to enter on the keyboard his or her ratings of another 10 or so pairings for practice. When the subject understood the instructions and mastered the task, the actual experimental session, which took about 40 min per subject, commenced.

\section{RESULTS}

\section{Individual Differences}

In our previous study on flat rectangles, we found that the contrast between the two main design diagonal ratings, $d(3,7)$ and $d(1,9)$ (see Schönemann et al., 1985, Figure 4c) sufficed to classify the subjects into three wellseparated strategy groups. We classified the subjects into the predominantly shape group (labeled UP in the previous paper) when this index exceeded 1.5, into the predominantly area group (DWN) when it was less than -1.5 , and into the HW group (EQ) when it was close to zero. The inclusion of tall rectangles renders this 1-D index ineffective, because it fails to discriminate between

Table 1

Scatterplot of Area Diagonals and Shape Diagonals Used As Discriminators for Defining Strategy Groups

\begin{tabular}{|c|c|c|c|c|c|c|c|c|c|c|c|}
\hline \multirow{2}{*}{$\begin{array}{c}d(1,16) \\
\text { Area Diagonal } \\
\end{array}$} & \multicolumn{11}{|c|}{$d(4,13)$ Shape Diagonal } \\
\hline & 0 & 1 & 2 & 3 & 4 & 5 & 6 & 7 & 8 & 5 & $\overline{\text { Frequencies }}$ \\
\hline 9 & & 3 & 1 & & & & & 1 & 3 & 2 & 10 \\
\hline 8 & & 2 & 1 & & & & & 1 & 3 & & 7 \\
\hline 7 & & 2 & 3 & & & & 2 & 2 & & & 9 \\
\hline 6 & 1 & 1 & & & 1 & 2 & & & & & 5 \\
\hline 5 & & & 1 & 1 & & & & & & & 2 \\
\hline 4 & & $g$ & oup & 1 & & & IW & gr & & & 2 \\
\hline 3 & & & & & & & & & & & 0 \\
\hline 2 & 2 & 1 & & & & & & & & & 3 \\
\hline 1 & 2 & & 2 & & & & & & & & 4 \\
\hline 0 & 1 & & gro & & & & & & & & 1 \\
\hline Frequencies & 6 & 9 & 8 & 2 & 1 & 2 & 2 & 4 & 6 & & 43 \\
\hline
\end{tabular}

Note-Boldface indicates the 4 subjects on the boundaries whom we dropped from the subgroup analyses. 
the subjects for whom both diagonal dissimilarities are large and the subjects for whom both are small. We therefore used a 2-D discriminant function to classify subjects into relatively homogeneous strategy subgroups, namely the pair, $d(4,13)$ (maximal shape difference) and $d(1,16)$ (maximal size difference, see Figure 1).

The resulting scatterplot (see Table 1) shows three wellseparated subgroups. Their separation is also reflected in the clearly bimodal marginal distributions. Although retaining the 4 subjects whose results fell on the boundaries would not have substantially altered the results, we decided to drop them, leaving 39 subjects, whom we classified into five subgroups:

HW1 group: $d(4,13)>4, d(1,16)>3 \quad 8$ subjects HW2 group: same as HW1 8 subjects

AX1 group: $d(4,13)<4, d(1,16)>3 \quad 8$ subjects AX2 group: same as $\mathrm{AX} 1 \quad 7$ subjects

$\mathrm{X}$ group: $\quad d(4,13)<4, d(1,16)<3 \quad 8$ subjects

The reason we partitioned the larger strategy groups was to allow cross-validation of the numerical estimates over two independent subgroups in order to gauge the robustness of the estimates across replications. Since the $X$ group was rather small, it seemed unwise to subdivide it further. The average relative dissimilarities for the three major strategy groups are listed in Table 2 . To obtain the observed group averages, these figures should be multiplied by 9 .

An intuitive interpretation of the three strategy groups is apparent at this stage without detailed numerical analysis. For the HW groups, both diagonal ratings, $d(1,16)$ and $d(4,13)$, are large and equal, which means that subjects in these groups preserved the orthogonality of the original design by using height and width roughly equally and, therefore, discriminated among all 16 stimuli. Because, for the AX groups, the shape diagonal had a much smaller average length than the area diagonal, the AX subjects treated tall and flat rectangles of the same eccentricity as equivalent, thus collapsing the design along the area diagonal. Finally, because the subjects in the $\mathrm{X}$ group assigned small ratings along both diagonals of the design, they collapsed the design still further by also discounting area, thus working with a very small quotient set of discriminable stimuli.

Table 2

Relative Average Dissimilarities for the Three Major Strategy Groups

\begin{tabular}{|c|c|c|c|c|c|c|c|c|c|c|c|c|c|c|c|c|}
\hline \multicolumn{17}{|c|}{ Below Diagonal: HW Groups $(N=18)$, Above Diagonal: AX Groups $(N=17)$} \\
\hline & 1 & 2 & 3 & 4 & 5 & 6 & 7 & 8 & 9 & 10 & 11 & 12 & 13 & 14 & 15 & 16 \\
\hline 1 & .00 & .45 & .58 & 67 & .42 & .48 & .75 & .83 & .54 & .74 & .69 & .87 & .67 & .82 & .83 & .85 \\
\hline 2 & .42 & .00 & .31 & .45 & .15 & .48 & .56 & .62 & .29 & .61 & .74 & .74 & .50 & .68 & .85 & .89 \\
\hline 3 & .59 & .33 & .00 & .31 & .47 & .63 & .47 & .54 & .20 & .66 & .67 & .76 & .21 & .67 & .72 & .87 \\
\hline 4 & .76 & .49 & .31 & .00 & .62 & .67 & .57 & .43 & .40 & .67 & .77 & .62 & .17 & .66 & .75 & .73 \\
\hline 5 & .25 & .52 & .66 & .77 & .00 & .47 & .61 & .71 & .31 & .49 & .76 & .82 & .44 & .60 & .76 & .87 \\
\hline 6 & .48 & .31 & .47 & .59 & .47 & .00 & .42 & .61 & .61 & .40 & .45 & .61 & .67 & .56 & .61 & .62 \\
\hline 7 & .73 & .42 & .31 & .42 & .66 & .34 & .00 & .34 & .54 & .26 & .40 & .46 & .61 & .33 & .57 & .66 \\
\hline 8 & .79 & .55 & .39 & .32 & .74 & .58 & .36 & .00 & .63 & .50 & .55 & .33 & .54 & .20 & .56 & .57 \\
\hline 9 & .36 & .63 & .71 & .81 & .26 & .58 & .68 & .82 & .00 & .49 & .67 & .75 & .31 & .53 & .76 & .86 \\
\hline 10 & .66 & .49 & .54 & .66 & .50 & .34 & .44 & .70 & .51 & .00 & .43 & .59 & .61 & .39 & .42 & .62 \\
\hline 11 & .75 & .55 & .46 & .58 & .70 & .41 & .33 & .45 & .71 & .47 & .00 & .37 & .78 & .54 & .35 & .43 \\
\hline 12 & .88 & .65 & .58 & .50 & .84 & .60 & .46 & .33 & .79 & .62 & .35 & .00 & .72 & .50 & .25 & .36 \\
\hline 13 & .47 & .76 & .79 & .85 & .39 & .77 & .77 & .84 & .27 & .55 & .77 & .83 & .00 & .45 & .67 & .81 \\
\hline 14 & .79 & .61 & .64 & .76 & .69 & .47 & .58 & .60 & .56 & .28 & .52 & .64 & .53 & .00 & .48 & .60 \\
\hline 15 & .85 & .69 & .64 & .70 & .79 & .56 & .53 & .56 & .76 & .49 & .37 & .49 & .70 & .41 & .00 & .39 \\
\hline 16 & .89 & .78 & .67 & .66 & .91 & .70 & .62 & .49 & .90 & .69 & .40 & .36 & .84 & .68 & .41 & .00 \\
\hline 1 & .00 & & & & & & & & & & & & & & & \\
\hline 2 & .52 & .00 & & & & & & & & & & & & & & \\
\hline 3 & .73 & .37 & .00 & & & & & & & & & & & & & \\
\hline 4 & .72 & .40 & .38 & .00 & & & & & & & & & & & & \\
\hline 5 & .63 & .13 & .46 & .60 & .00 & & & & & & & & & & & \\
\hline 6 & .27 & .65 & .64 & .77 & .60 & .00 & & & & & & & Group & $(N=$ & & \\
\hline 7 & .61 & .36 & .50 & .52 & .45 & .53 & .00 & & & & & & & & & \\
\hline 8 & .68 & .33 & .40 & .46 & .53 & .68 & .42 & .00 & & & & & & & & \\
\hline 9 & .66 & .30 & .13 & .48 & .41 & .71 & .51 & .41 & .00 & & & & & & & \\
\hline 10 & .60 & .56 & .55 & .66 & .40 & .56 & .14 & .53 & .43 & .00 & & & & & & \\
\hline 11 & .16 & .67 & .63 & .74 & .64 & .19 & .49 & .62 & .69 & .51 & .00 & & & & & \\
\hline 12 & .55 & .44 & .53 & .59 & .48 & .51 & .37 & .41 & .63 & .37 & .57 & .00 & & & & \\
\hline 13 & .69 & .52 & .31 & .10 & .47 & .75 & .55 & .49 & .37 & .59 & .76 & .61 & .00 & & & \\
\hline 14 & .71 & .63 & .53 & .55 & .33 & .63 & .38 & .13 & .38 & .41 & .62 & .38 & .45 & .00 & & \\
\hline 15 & .58 & .61 & .71 & .68 & .36 & .53 & .52 & .54 & .59 & .31 & .47 & .11 & .60 & .46 & .00 & \\
\hline 16 & .15 & .71 & .79 & .73 & .75 & 19 & .55 & .66 & .71 & .43 & .31 & .60 & .71 & .63 & .55 & .00 \\
\hline
\end{tabular}

Note-Boldface indicates dissimiarlity ratings for squares, which are much smaller for the $\mathrm{X}$ group than for the other two strategy groups. 


\section{Within-Subject Analyses: Metric Problem}

As in Schönemann et al. (1985), we fitted several different composition rules, $c=g(a, b)$, that might account for the subadditivity, and evaluated the fit in terms of both mean residuals (bias) and sums of squared residuals (actual fit):

$\begin{array}{lrl}\text { CB } & := & a+b: \text { city-block metric. } \\ \text { SUP } & := & \max \{a, b\}: \text { supremum metric. } \\ \text { EUC }:= & \left(a^{2}+b^{2}\right)^{1 / 2}: \text { Euclidean metric, except } \\ & \text { that this composition is also applied } \\ & \text { within dimensions, so that it becomes } \\ & \text { segmentally subadditive. } \\ \operatorname{MBR}(L):= & (a+b) /(1+a b), \text { where } L=9, \text { is the up- } \\ & \text { per bound of the response scale used to } \\ & \text { compute the relative dissimilarities, } \\ & d=d^{*} / L, \text { from the observed values, } d^{*} . \\ \operatorname{MBR}(s L):= & \text { same as MBR }(L), \text { except that the bound } \\ & \text { is reduced to } s L, \text { with } s=.8 . \\ \operatorname{MBR}(M X):= & \text { same as MBR }(L), \text { except that the actual } \\ & \text { upper bound, } L, \text { is replaced by the max- } \\ & \text { imal response of the subject, thus giv- } \\ & \text { ing rise to an individual-difference } \\ & \text { metric. }\end{array}$

See Schönemann et al. (1985) for a more detailed discussion of the rationale for choosing these metrics, and some of their formal properties.

Table 3a

Residual Means (Bias) and Sums of Squares (Fit) for Various Subadditive Concatenations $g$

\begin{tabular}{|c|c|c|c|c|c|c|c|c|}
\hline Group & $\mathrm{CB}$ & SUP & EUC & $\mathbf{M}(L)$ & $\mathrm{M}(M X)$ & $\mathrm{M}(s L)$ & AVER & AVPW \\
\hline \multicolumn{9}{|c|}{ Height } \\
\hline HW1 & + & - & & & & & - & \\
\hline HW2 & + & - & & & & & - & \\
\hline AX1 & + & - & & & & & & \\
\hline $\mathrm{AX} 2$ & + & - & & & & & - & \\
\hline $\mathrm{X}$ & + & & & + & & & - & \\
\hline \multicolumn{9}{|c|}{ Width } \\
\hline HW1 & + & & & & & & - & \\
\hline HW2 & + & - & & & & & - & \\
\hline AX1 & + & - & & & & & - & \\
\hline $\mathrm{AX} 2$ & + & - & & & & & & \\
\hline $\mathbf{X}$ & + & & + & + & & & - & \\
\hline \multicolumn{9}{|c|}{ Shape Diagonal } \\
\hline HW1 & + & - & & & & & - & \\
\hline HW2 & + & & & + & + & & - & \\
\hline AX1 & + & + & + & + & + & + & + & + \\
\hline $\mathrm{AX} 2$ & + & + & + & + & + & + & + & + \\
\hline $\mathbf{X}$ & + & + & + & + & + & + & + & + \\
\hline \multicolumn{9}{|c|}{ Area Diagonal } \\
\hline HW1 & + & - & & & & & - & \\
\hline HW2 & + & - & & & & & - & \\
\hline $\mathbf{A X 1}$ & + & - & - & & & & - & \\
\hline $\mathbf{X}$ & + & - & & & & & - & \\
\hline
\end{tabular}

Note-Bias patterns of means for residuals $f=a+b-c$ : blank : $=$ mean $f$ between -.1 and $.1 ;-:=$ mean $f<-.1$ ( $g$ too subadditive for data); + := mean $f>.1$ ( $g$ not subadditive enough). The symbol MBR has been shortened to $M$.
The results of these comparisons for collinear triples parallel to the design coordinates, height and width, and along both diagonals of the design, area and shape (Tables 3a and 3b), are virtually identical to the results we reported in Schönemann et al. (1985, Tables 2-4).

We again found a substantial positive bias for the cityblock metric across all groups and all comparisons, measuring roughly .2 on a $(0,1)$ scale, which confirms that these new data are consistently subadditive (Table $3 a$ ). The averaging function is again consistently biased in the opposite direction: it is too subadditive for these data. A new result is that all eight functions leave a positive bias along the shape diagonal $(4,13)$ in Table $3 a$. In our previous study, we did not distinguish between the two design diagonals. Thus, triples along the shape diagonal give rise to the most subadditive ratings. In terms of bias alone, $\operatorname{MBR}(s L)$ and AVPW tie for first place.

The actual fit is tabulated relative to the sums of squares left by the strictly additive city-block metric in Table $3 \mathrm{~b}$. An overall summary of the fit across the various subgroups is given in the bottom row of this table. The MBR with a reduced upper bound [MBR(sL) with $s L=7.2]$ does much better than the average power rule, and also better than the simpler $\operatorname{MBR}(L)$, which does not do particularly well at the within-subject level. However, we found in our previous study (Schönemann et al., 1985) that this relative advantage of the $\operatorname{MBR}(s L)$ versus the $\operatorname{MBR}(L)$ does not hold for subgroup averages.

We conclude that the inverse hyperbolic tangent transformation removes most of the subadditivity in the observed ratings.

\section{Psychophysical Problem}

At this point in our previous report (Schönemann et al., 1985), we addressed the orientation problem, that is, the problem of whether the subjects used a subjective $\mathrm{H} / \mathrm{W}$ or an $\mathrm{A} / \mathrm{S}$ coordinate system. We were unable to resolve this problem conclusively on the basis of the withinsubjects results. The MDS analyses of the subgroup averages suggested, not surprisingly, that the HW group used height and width, and that the two other groups used area and shape with differing emphases. However, these inferences also remained tentative at the subgroup level, because the differences between the numerical indices of fit were small and inconclusive.

The inclusion of squares in our new design permits us to approach this problem more directly by reformulating it as a psychophysical problem: If we can relate the physical dimensions of the stimuli, for example, height and width of the rectangles in centimeters, directly to the average dissimilarities in each subgroup, we can predict the outcome of the MDS analyses by simply plotting the stimuli in the physical dimensions each strategy group employed without any need for subjective coordinates.

Our previous study (Schönemann et al., 1985) and the within-subject results of our new data suggest that these psychophysical maps will be of the form

$$
d_{i j}=k \tanh \left[h\left(H_{i}, H_{j}, W_{i}, W_{j}\right)\right] .
$$


Table 3b

Residual SSQ Relative to SSQ Left by the City-Block Metric

\begin{tabular}{|c|c|c|c|c|c|c|c|c|c|c|}
\hline Group & CB & SUP & EUC & $\mathrm{M}(L)$ & $\mathrm{M}(M X)$ & $\mathrm{M}(s L)$ & AVER & AVPW & SSCB & $n$ \\
\hline \multicolumn{11}{|c|}{ Height } \\
\hline HW1 & 1.0 & .56 & .28 & .14 & .13 & .21 & 1.07 & .27 & .09 & 64 \\
\hline HW2 & 1.0 & .34 & .26 & .20 & .19 & .17 & .58 & .19 & .12 & 64 \\
\hline AX1 & 1.0 & .26 & .18 & .17 & .14 & .09 & .57 & .12 & .10 & 64 \\
\hline $\mathrm{AX} 2$ & 1.0 & .29 & .18 & .14 & .14 & .16 & .54 & .16 & .12 & 56 \\
\hline $\mathrm{x}$ & 1.0 & .21 & .33 & .21 & .17 & .14 & .31 & .18 & .28 & 64 \\
\hline \multicolumn{11}{|c|}{ Width } \\
\hline HW1 & 1.0 & .47 & .32 & .43 & .41 & .32 & .81 & .50 & .04 & 64 \\
\hline HW2 & 1.0 & .42 & .30 & .30 & .28 & .22 & .68 & .20 & .09 & 64 \\
\hline AX1 & 1.0 & .41 & .22 & .26 & .24 & .17 & .66 & .17 & .08 & 64 \\
\hline AX2 & 1.0 & .33 & .23 & .17 & .16 & .14 & . .53 & .14 & .12 & 56 \\
\hline $\mathbf{X}$ & 1.0 & .14 & .27 & .16 & .12 & .09 & .20 & .13 & .29 & 64 \\
\hline \multicolumn{11}{|c|}{ Shape Diagonal } \\
\hline HW1 & 1.0 & .56 & .18 & .16 & .15 & .25 & 1.19 & .32 & .07 & 48 \\
\hline HW2 & 1.0 & .20 & .25 & .19 & .18 & .10 & .27 & .12 & .24 & 48 \\
\hline $\mathbf{A X 1}$ & 1.0 & .19 & .42 & .40 & .36 & .27 & .18 & .30 & .73 & 48 \\
\hline $\mathrm{AX2}$ & 1.0 & .23 & .45 & .33 & .32 & .22 & .22 & .29 & .97 & 42 \\
\hline $\mathbf{X}$ & 1.0 & .25 & .47 & .41 & .37 & .29 & .23 & .33 & .93 & 48 \\
\hline \multicolumn{11}{|c|}{ Area Diagonal } \\
\hline HW1 & 1.0 & .59 & .23 & .19 & .17 & .28 & 1.42 & .44 & .07 & 48 \\
\hline HW2 & 1.0 & .86 & .40 & .25 & .22 & .26 & 1.35 & .30 & .08 & 48 \\
\hline $\mathbf{A X} 1$ & 1.0 & 1.65 & .56 & .28 & .27 & .42 & 2.60 & .48 & .04 & 48 \\
\hline $\mathrm{AX} 2$ & 1.0 & .22 & .24 & .09 & .09 & .09 & .31 & .09 & .26 & 42 \\
\hline $\mathrm{X}$ & 1.0 & .33 & .47 & .49 & .43 & .37 & .17 & .39 & .21 & 48 \\
\hline
\end{tabular}

Number of Times Each Metric Placed Among First Three in Each Row

$\begin{array}{llllllll}0 & 4 & 4 & 7 & 15 & 17 & 4 & 9\end{array}$

\begin{abstract}
Note-Entries are ratios of residual SSQ $g(a, b)] / \mathrm{SSQ}[\mathrm{CB}]$, where $g$ is one of the eight metrics described in the text, indicating the degree to which the metric $g$ fits better than the strictly additive CB. For example, if the entry is .2, the metric fits 5 times better than the CB. The absolute (mean) SSQ for the CB are given in the column SSCB for relative distances, that is, on a scale from 0 to 1 . All other absolute SSQ can be found by multiplying the ratios in the table by this value. Proper fractions indicate that the particular metrics fit better than the CB. $n:=$ sample size. The symbol MBR has been shortened to $M$.
\end{abstract}

The constant $k$ can be set to $L=9$ in all groups, because we know already, from our previous analyses, that the small advantage of the MBR with reduced upper bound, $\operatorname{MBF}(s L)$, over $\operatorname{MBR}(L)$ at the within-subject level does not carry over to averaged data. In fact, we found that the simpler $\operatorname{MBR}(L)$ gave slightly better fits than MBR(sL) for the group averages (Schönemann et al., 1985, Table 6, Part C). We also know already that $h$ will be different for each of the three subgroups. Thus, the psychophysical problem reduces to a search for an explicit expression for $h$ in each of the three strategy groups. This search will be easiest for the squares, because they vary only along one dimension.

\section{Psychophysical Maps for Squares}

Because we expect the psychophysical maps to be different in each strategy group, the following analyses were performed for all five groups separately.

To find the psychophysical maps for squares, we extracted the six collinear ratings along the minor diagonal of the design: $d(1,6), d(6,11), d(11,16), d(1,11)$, $d(6,16), d(1,16)$. After converting them into relative distances by dividing by the upper bound of 9 , we applied Fisher's Z-transformation to these relative distances. The result is a skew-symmetric matrix, $C^{*}=-C^{*}$, which we treat as an estimate of the 1-D scaling model

$$
\begin{aligned}
C=\left(c_{i j}\right) & =-C^{\prime}, \\
& \text { with } c_{i j}:=\tanh ^{-1}\left(d^{*}{ }_{i j} / 9\right)=u_{i}-u_{j},
\end{aligned}
$$

where the $d^{*}{ }_{i j}$ are oriented MBR distances and the $u_{i}$, hyperbolic angles. This model is the 1-D analogue of the MBR (Equation 4; see Appendix) which defines the scale value $u_{i}$ up to a translation. It is well known that in the fallible case the least squares estimates of the $u_{i}$ are given by the column means of $C^{*}$ (see, e.g., Schönemann, 1985).

The rationale for invoking Equation 7 is that dissimilarity ratings of squares can depend on only one subjective dimension, "size," because squares vary along only one physical dimension. However, this dimension is not uniquely defined: width, area, the length of either diagonal, the log of these lengths, or any other bijective (1:1 onto) transform of physical width in centimeters, are all equivalent physical characterizations of square stimuli. Thus, two questions arise: (1) How well does Equation 7 fit? and (2) If it does fit, what physical definition of the squares bears the simplest relation to the subjective scale values? 
Table 4

Data Base for Psychophysical Maps for Squares

\begin{tabular}{|c|c|c|c|c|c|c|c|c|c|c|c|c|c|c|c|c|}
\hline \multirow[b]{2}{*}{ Group } & \multicolumn{6}{|c|}{$\begin{array}{c}\text { Observed Relative } \\
\text { Dissimilarities }\end{array}$} & \multicolumn{4}{|c|}{ Scale Values } & \multicolumn{6}{|c|}{ Residuals } \\
\hline & 12 & 13 & 14 & 23 & 24 & 34 & $u_{1}$ & $u_{2}$ & $u_{3}$ & $u_{4}$ & 12 & 13 & 14 & 23 & 24 & 34 \\
\hline $\begin{array}{l}\text { HW1 } \\
\text { HW2 }\end{array}$ & $\begin{array}{l}56 \\
40\end{array}$ & $\begin{array}{l}78 \\
72\end{array}$ & $\begin{array}{l}93 \\
85\end{array}$ & $\begin{array}{l}39 \\
43\end{array}$ & $\begin{array}{l}67 \\
73\end{array}$ & $\begin{array}{l}41 \\
40\end{array}$ & $\begin{array}{l}-83 \\
-64\end{array}$ & $\begin{array}{l}-15 \\
-24\end{array}$ & $\begin{array}{l}25 \\
24\end{array}$ & $\begin{array}{l}73 \\
64\end{array}$ & $\begin{array}{r}08 \\
-10\end{array}$ & $\begin{array}{l}00 \\
03\end{array}$ & $\begin{array}{r}02 \\
-01\end{array}$ & $\begin{array}{r}-09 \\
03\end{array}$ & $\begin{array}{r}-11 \\
03\end{array}$ & $\begin{array}{l}-07 \\
-10\end{array}$ \\
\hline $\begin{array}{l}\mathrm{AX1} \\
\mathrm{AX} 2\end{array}$ & $\begin{array}{l}41 \\
56\end{array}$ & $\begin{array}{l}65 \\
74\end{array}$ & $\begin{array}{l}85 \\
85\end{array}$ & $\begin{array}{l}37 \\
55\end{array}$ & $\begin{array}{l}59 \\
65\end{array}$ & $\begin{array}{l}33 \\
55\end{array}$ & $\begin{array}{l}-62 \\
-72\end{array}$ & $\begin{array}{l}-15 \\
-19\end{array}$ & $\begin{array}{l}20 \\
24\end{array}$ & $\begin{array}{l}57 \\
67\end{array}$ & $\begin{array}{l}04 \\
13\end{array}$ & $\begin{array}{r}-01 \\
02\end{array}$ & $\begin{array}{r}02 \\
-03\end{array}$ & $\begin{array}{r}-01 \\
12\end{array}$ & $\begin{array}{l}-07 \\
-07\end{array}$ & $\begin{array}{r}-04 \\
12\end{array}$ \\
\hline $\mathbf{X}$ & 27 & 16 & 15 & 19 & 19 & 31 & -15 & -03 & 01 & 17 & 17 & -05 & -15 & 09 & -02 & 21 \\
\hline
\end{tabular}

The scale values $u_{i}$ (translated so that they sum to zero) and the residuals are shown in Table 4 . The relations between these scale values and the widths of the squares in centimeters are depicted in Figure 2 . There is evidently no need to search further for a simple psychophysical map relating width to the $u_{i}$ values, because the relation between the subjective size of squares and their physical width is virtually linear (with a very slight quadratic bend) in both HW groups. This enables us to discard the subjective $u_{i}$ values altogether and to replace them with physical width in centimeters. Thus, the function $h$ in Equation 6 is linear for squares.

Similar results are obtained in the two AX groups, but those of the $\mathrm{X}$ group appear drastically different. On fitting the linear model with metric width differences in centimeters to the $c^{*}{ }_{i j}$ in Equation 7 as dependent variables, we arrived at the following psychophysical maps for the subset of the four squares:

$$
\begin{aligned}
& \text { HW1 group: } d=9 \tanh \left[.26\left(W_{1}-W_{\mathrm{s}}\right)\right] \\
& \text { HW2 group: } d=9 \tanh \left[.22\left(W_{1}-W_{\mathrm{s}}\right)\right] \\
& \text { AX1 group: } d=9 \tanh \left[.20\left(W_{1}-W_{\mathrm{s}}\right)\right] \\
& \text { AX2 group: } d=9 \tanh \left[.22\left(W_{1}-W_{\mathrm{s}}\right)\right] \\
& \text { X group: } \quad d=9 \tanh \left[.03\left(W_{1}-W_{\mathrm{s}}\right)\right]
\end{aligned}
$$

where $W_{1}:=\max \left\{W_{i}, W_{j}\right\}, H_{\mathrm{s}}:=\min \left\{H_{i}, H_{\mathrm{j}}\right\}$, and so forth, that is, the subscripts $s$ and 1 stand for "smaller" and "larger." This notation ensures that the indicated differences are always nonnegative so that absolute value signs can be avoided. The parameter estimates are relative to the physical units in centimeters.

Because the slopes in the first four groups are virtually identical, we conclude (1) that these subjects did not base their dissimilarity judgments of squares on physical area, as one might have thought, but rather on linear extent of the sides, and (2) that the slope of the width differences that enter the tanh transformation as an argument is the same for the HW and AX subjects, roughly of the order of .2 .

The subjects in the last, $X$, group were clearly different from those in the other four groups in their virtually ignoring size, that is, they projected all four squares into one equivalence class.

\section{Psychophysical Maps for Rectangles}

We now ask whether these results generalize to rectangles that are not square. To answer this, we investigated the simplest possibility first, which is that subjects in the HW group continued to use linear extent along both height and width, rather than some more complicated function $h$ as an argument for the tanh transformation, which preserves the upper bound.

To test this hypothesis, we again fitted the linear model to the Fisher $Z$-transformations of the relative distances, with $w_{1}, w_{s}, h_{1}$ and $h_{s}$ as independent variables. We arrived at the following psychophysical maps for the two HW groups:

HW1 group:

$$
d=9 \tanh \left[.09 W_{1}-.04 W_{\mathrm{s}}+.16 H_{\mathrm{l}}-.19 H_{\mathrm{s}}\right]
$$

HW2 group:

$$
d=9 \tanh \left[.10 W_{1}-.07 W_{\mathrm{s}}+.11 H_{1}-.10 H_{\mathrm{s}}\right] .
$$
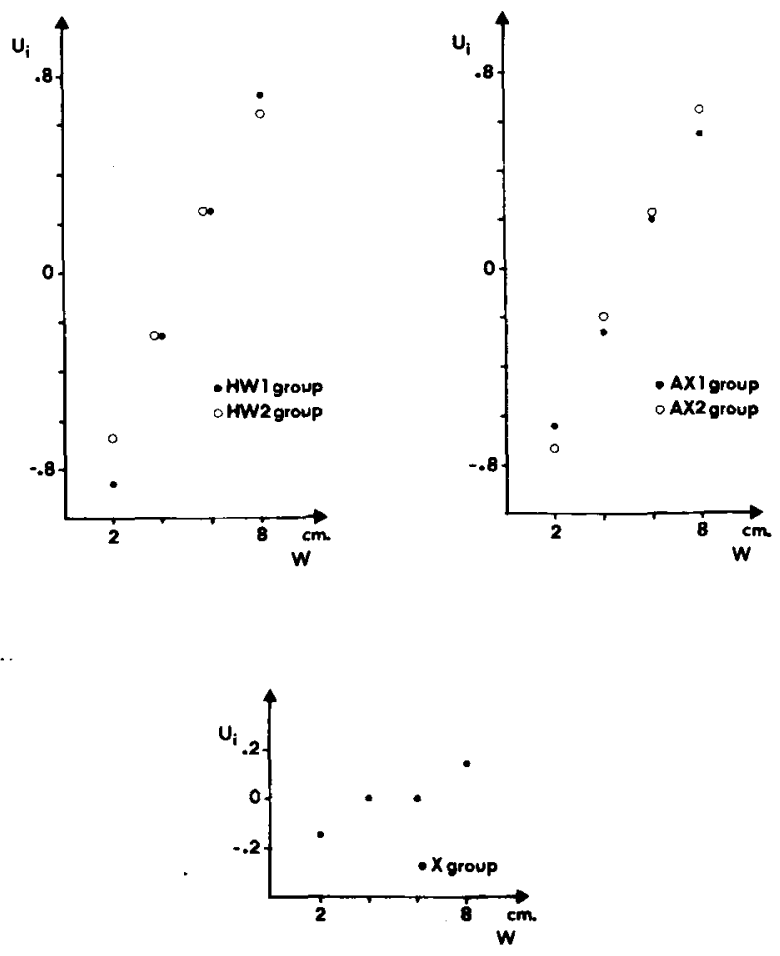

Figure 2. Subjective versus physical size of squares. Relation between physical width in and subjective size for the five groups. Physical width is defined in centimeters, subjective size by $u$ in the scaling model (Equation 7). 
To find the corresponding maps for the two AX groups, we recall that the diagonal ratings, $d(4,13)$ and $d(1,16)$, suggested that subjects in these groups tended to collapse the design along the area diagonal, thus folding the shape diagonal at its midpoint. We call the resulting dimension "eccentricity," because it indicates the degree of departure from squareness. Encouraged by our results with the two HW groups, we again began with the simplest possible hypothesis, which is that eccentricity is proportional to the magnitude of the difference between height and width. Similarly, if the linear relation between subjective size and physical width that we found for squares (Equations 8a-8e) generalizes to rectangles, then, as a first approximation, subjective "area" of rectangles might be proportional to the average of height and width. These conjectures suggest the following independent variables for the AX group:

$$
\begin{array}{ll}
\text { (physical) "area" } & \mathrm{A}:=(H+W) / 2, \\
\text { (physical) "eccentricity" } \mathrm{X}:=|H-W| / 2 .
\end{array}
$$

On entering their values into the design matrix and fitting this linear model to the Fisher Z-transformations of the relative distances for the two $\mathrm{AX}$ groups, we arrived at the following psychophysical maps for the two $\mathrm{AX}$ groups:

AX1 group:

$$
d=9 \tanh \left[.15 X_{1}-.11 X_{\mathrm{s}}+.17 A_{1}-.15 A_{\mathrm{s}}\right]
$$

AX2 group:

$$
d=9 \tanh \left[.12 X_{1}-.09 X_{\mathrm{s}}+.23 A_{1}-.20 A_{\mathrm{s}}\right]
$$

Finally, for the $\mathrm{X}$ group, both diagonal indices, $d(4,13)$ and $d(1,16)$, were small, which suggests that subjects in this group discarded area differences altogether and based their discriminations on eccentricity alone. An obvious way to check this conjecture is to fit the above AX model to the data of the $X$ group. Under the hypothesis we expect nonzero weights for the two $X$ arguments and zero weights for the $A$ arguments. The estimates we obtained,

$\mathrm{X}$ group:

$$
d=9 \tanh \left[.22 X_{1}-.23 X_{\mathrm{s}}+.08 A_{1}-.03 A_{\mathrm{s}}\right],
$$

support this prediction up to a point, although some small area weights remain. On refitting the linear model with eccentricity alone, we arrived at the two-parameter psychophysical map:

$$
\mathrm{X} \text { group: } d=9 \tanh \left[.37 X_{1}-.24 X_{s}\right] \text {. }
$$

In response to a reviewer's query, we emphasize that Equations 8-12 are all versions of the MBR, Equation 4.

\section{Evaluation of Fit}

We briefly pause to check to what extent the psychophysical maps above fit within each sample, although we consider it more important that they evidently replicate across independent samples within strategy groups.
Because the parameter estimates of the five psychophysical maps were obtained with the general linear model fitted to the Fisher Z-transformations of the observed relative distances, the most obvious measure for assessing the fit would seem to be

$$
\text { eta }^{2}:=\frac{\text { between sum of squares }}{\text { total sum of squares }}
$$

of the dependent variables, $c^{*}{ }_{i j}:=\tanh ^{-1}(d / 9)$. A somewhat more indirect index of fit is the correlation $r$ between the independent variables and the predictions from the psychophysical maps. Eta and $r$ are not entirely equivalent figures of merit. In the present context, we consider eta the more appropriate index. Standard significance tests are ruled out because neither $r$ nor eta are based on independent observations.

The values for the three fit indices, eta ${ }^{2}$, eta, and $r$, for the above and some subsequent maps are collected in Table 5 . The average eta ${ }^{2}$ across all five groups was .960 , with an average eta of .980 . The average correlation was .856. Thus, on the basis of these indices, the five psychophysical functions appear to describe the data quite well within each group. In each of the first four groups, we describe 120 observations with maps that depend on only four parameters. The 120 observations of the $X$ group are described with a map that requires only two parameter estimates.

Overall, then, we conclude from the within-group fit indices and the between-group comparisons that the psychophysical maps for the three strategy groups are adequate and relatively stable descriptions of the judgment functions employed by the subjects in each strategy group.

\section{Reduced Approximations}

We now ask how much the fit deteriorates in each group if we approximate the four-parameter maps with maps that

\begin{tabular}{|c|c|c|c|c|c|c|c|c|c|}
\hline \multirow[b]{2}{*}{ Group } & \multicolumn{3}{|c|}{$\begin{array}{c}\text { Four- } \\
\text { Parameter Maps }\end{array}$} & \multicolumn{3}{|c|}{$\begin{array}{l}\text { Unweighted One- } \\
\text { Parameter Maps }\end{array}$} & \multicolumn{3}{|c|}{$\begin{array}{l}\text { Weighted One- } \\
\text { Parameter Maps }\end{array}$} \\
\hline & $\mathrm{eta}^{2}$ & eta & $r$ & eta $^{2}$ & eta & $r$ & $\overline{\text { eta }^{2}}$ & eta & $r$ \\
\hline$\overline{\text { HW1 }}$ & .97 & .98 & .92 & .79 & .89 & .78 & .89 & .95 & .73 \\
\hline HW2 & .98 & .99 & .92 & .52 & .72 & .90 & .98 & .99 & .90 \\
\hline AX1 & .95 & .98 & .82 & .50 & .71 & .83 & .83 & .97 & .79 \\
\hline $\mathbf{A X 2}$ & .95 & .98 & .84 & .45 & .67 & .81 & .64 & .80 & .77 \\
\hline $\mathbf{X}$ & .95 & .97 & .78 & .56 & .75 & .60 & .86 & .93 & .66 \\
\hline Mean & .960 & .980 & .856 & .564 & .748 & .784 & .840 & .916 & .770 \\
\hline
\end{tabular}
depend on only one parameter, for example, in the simplest case, by hyperbolic tangent transformations of uniformly weighted linear combinations of the four arguments. This simple model predicts the observed dissimilarities as hyperbolic tangent transformations of the

Table 5

Fit Indices for Psychophysical Maps of Rectangles

Note-All fit indices are relative to $c_{l j}:=\tanh ^{-1}\left(d_{i j} / 9\right)$, where $d_{i j}$ are the 120 observed average dissimilarity ratings in each group (see Table 2). eta ${ }^{2}:=\operatorname{SSB} / \mathrm{SSW}, r:=$ correlation $\left(c_{i j}, c_{i j}\right)$, where the predicted $c_{i j}$ are computed from the physical stimulus coordinates in $\mathrm{cm}$ on the basis of the psychophysical maps (Equations 9-13 and 15-17) in the text. 
city-block metric computed from the metric design coordinates, that is, as $d=k \tanh (a+b)$, where $a$ is the magnitude of the width difference and $b$ is the magnitude of the height difference in centimeters. For each of the five groups, we found, as least squares estimates,

HW1 group: $d=9 \tanh \left[.12\left(W_{1}-W_{s}+H_{1}-H_{s}\right)\right]$,

HW2 group: $d=9 \tanh \left[.10\left(W_{1}-W_{\mathrm{s}}+H_{\mathrm{l}}-H_{\mathrm{s}}\right)\right]$,

AX1 group: $d=9 \tanh \left[.15\left(X_{1}-X_{\mathrm{s}}+A_{1}-A_{\mathrm{s}}\right)\right]$,

AX2 group: $d=9 \tanh \left[.16\left(X_{1}-X_{s}+A_{1}-A_{s}\right)\right]$,

$\mathrm{X}$ group: $\quad d=9 \tanh \left[.31\left(X_{1}-X_{\mathrm{s}}\right)\right]$,

with a reduction in average eta ${ }^{2}$ from .960 to .564 , in average eta from .980 to .748 , and in average $r$ from .856 to .784 . Thus, the reduction from four parameters to one has its price, although the fit still appears to be acceptable for data of this type. We will use these simple maps later to predict the outcome of MDS analyses.

Inspection of the parameter estimates of the fourparameter maps (Equations 9, 11, and 12) and the twoparameter maps (Equations 8 and 13) suggests a reason for the decline in eta $^{2}$ : With one exception, the weight attaching to the larger coordinate on each physical dimension exceeds that for the smaller coordinate. Thus, the fit should improve if the one-parameter approximations take this "saliency effect"' (Rosch, 1977) into account by applying fixed asymmetry weights (e.g., 1.2, -.8; the exact weight is probably not critical) to the larger and smaller arguments, so that again only one free parameter has to be estimated in each group. The least squares estimates for these weighted one-parameter maps are

HW1 group:

$$
d=9 \tanh \left[.08\left(1.2 W_{1}-.8 W_{\mathrm{s}}+1.2 H_{\mathrm{l}}-.8 H_{\mathrm{s}}\right)\right]
$$

HW2 group:

$$
d=9 \tanh \left[.07\left(1.2 W_{1}-.8 W_{\mathrm{s}}+1.2 H_{1}-.8 H_{\mathrm{s}}\right)\right]
$$

AX1 group:

$$
d=9 \tanh \left[.11\left(1.2 X_{1}-.8 X_{\mathrm{s}}+1.2 A_{\mathrm{I}}-.8 A_{\mathrm{s}}\right)\right]
$$

AX2 group:

$$
d=9 \tanh \left[.06\left(1.2 X_{1}-.8 X_{s}+1.2 A_{1}-.8 A_{s}\right)\right]
$$

$\mathrm{X}$ group: $d=9 \tanh \left[.31\left(1.2 X_{\mathrm{I}}-.8 X_{\mathrm{s}}\right)\right]$.

The prior weighting of the arguments raised the average eta ${ }^{2}$ from .564 to .840 and the average eta from .748 to .916 . It slightly lowered the average correlation $r$ from .784 to .770 . Because we put more trust into eta ${ }^{2}$ than into $r$, we conclude that weighting the arguments improves the fit of one-parameter psychometric maps sufficiently to treat them as reasonably close approximations to the four-parameter maps given earlier.

Finally, we consider an obvious compromise between one- and four-parameter maps to be two-parameter psychophysical maps that permit differential weighting of the relevant dimensions width, height, area, and shape.
Inspection of the four-parameter maps (Equations 9 and 11) suggests that such differential weighting will improve the fit, because both HW groups accentuate height over width, and the two AX groups area over shape (relative to the physical definitions of these dimensions). As least squares estimates for such two-parameter psychophysical maps we found:

HW1 group:

$$
d=9 \tanh \left[.04\left(1.2 W_{1}-.8 W_{\mathrm{s}}\right)+.12\left(1.2 H_{1}-.8 H_{\mathrm{s}}\right)\right],
$$

HW2 group:

$$
d=9 \tanh \left[.07\left(1.2 W_{1}-.8 W_{s}\right)+.09\left(1.2 H_{1}-.8 H_{s}\right)\right],
$$

AX1 group:

$$
d=9 \tanh \left[.09\left(1.2 X_{1}-.8 X_{\mathrm{s}}\right)+.13\left(1.2 A_{1}-.8 A_{\mathrm{s}}\right)\right],
$$

AX2 group:

$$
d=9 \tanh \left[.06\left(1.2 X_{1}-.8 X_{\mathrm{s}}\right)+.17\left(1.2 A_{1}-.8 A_{\mathrm{s}}\right)\right],
$$

with an average eta ${ }^{2}$ of .958 (eta $=.973$ ) and an average for $r$ of .825 across the above four groups. These twoparameter maps appear to strike an optimal compromise between parsimony and fit.

\section{MDS Analyses}

In principle, explicit knowledge of the psychophysical maps that relate physical coordinates to dissimilarities renders a multidimensional scaling analysis superfluous, because we can predict their outcome by simply plotting the stimuli in terms of the physical coordinates that enter each map through $h$ in Equation 6. However, the congruence will not be perfect, because we found that the fit could be improved by differentially weighting the larger and smaller coordinates, an adjustment not accounted for by the city-block metric. But we may expect its impact on the geometric representations to be slight because, in view of

$$
\begin{aligned}
& (1+c) Y_{1}-(1-c) Y_{\mathrm{s}} \\
& \quad=Y_{1}-Y_{\mathrm{s}}+c\left(Y_{1}+Y_{\mathrm{s}}\right)=\left|Y_{i}-Y_{j}\right|+c\left|Y_{i}+Y_{j}\right|,
\end{aligned}
$$

it affects only coordinates near 1 . Thus, as long as $c$ is small (we used $c=.2$ in Equations 16 and 17), the simpler unweighted one-parameter maps should be reasonably well in predicting the MDS configurations in each strategy group. ${ }^{2}$ This entails plotting the stimuli in the physical height/width system for the HW group, in the physical area/eccentricity system for the AX group, and along the eccentricity dimension for the $\mathrm{X}$ group. These plots are shown in Figures 3a, 3c, and 3e together with the actually obtained MDS configurations (see Note 1) based on the $\operatorname{MBR}(L)$ (Figures 3b, 3d, and 3f). Note that the MDS configuration for the $\mathrm{X}$ group, although clearly dominated by eccentricity, still reveals a weak area effect, in agreement with the four-parameter psychophysical map for this group (Equation 2). 


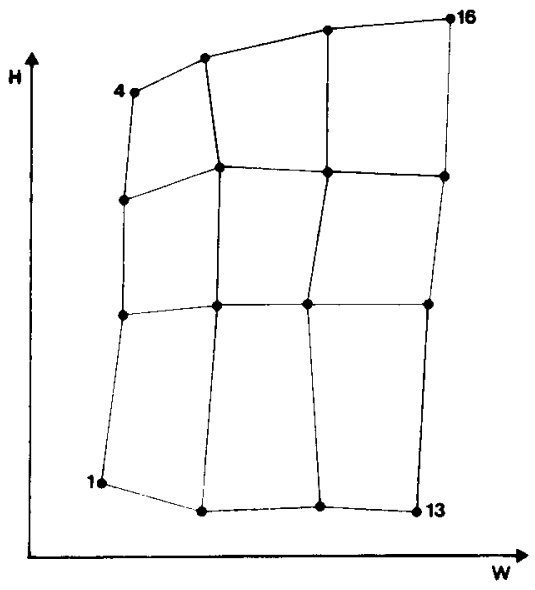

Fig. 3o

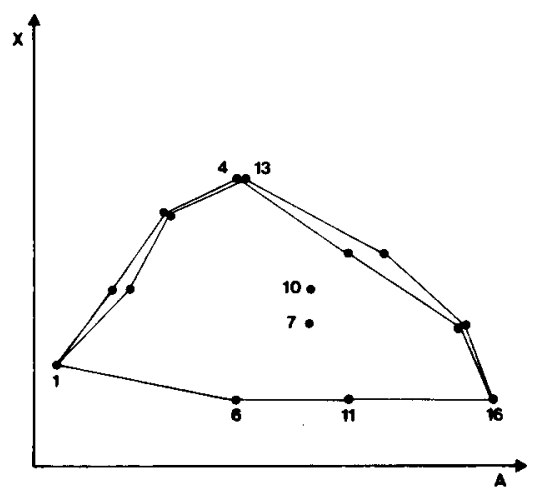

Fig. 3c

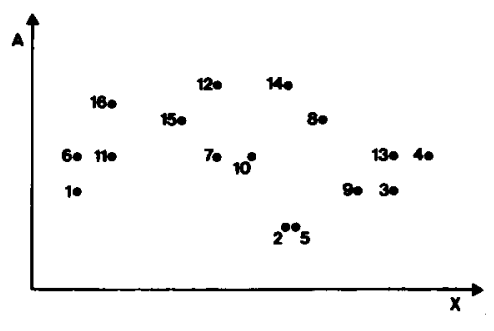

Fig. 3e

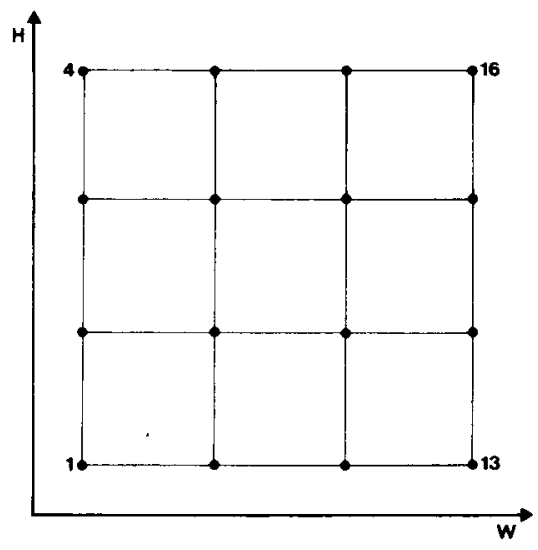

Fig. 3b

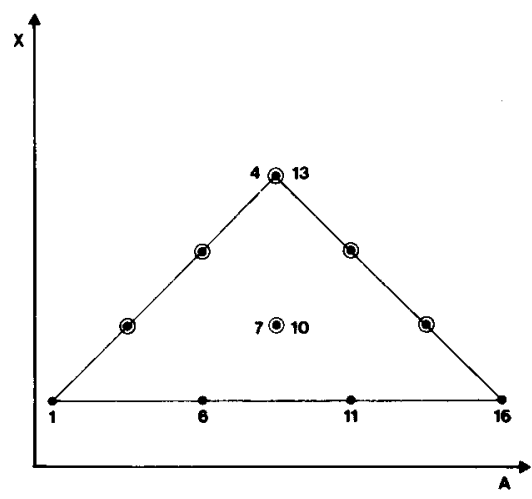

Fig. 3d

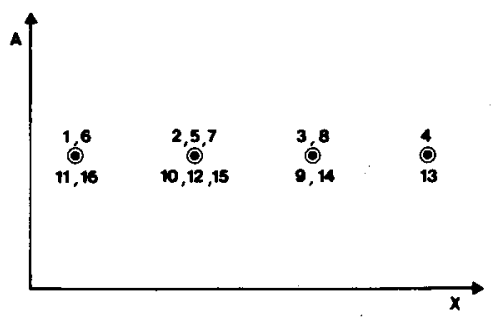

Fig.34

Figure 3. Predicted and observed MDS solutions: (a) Configuration predicted from physical height and width for HW group. (b) Observed configuration for HW group. (c) Configuration predicted from physical area and eccentricity for AX group. (d) Observed configuration for AX group. (e) Configuration predicted from physical eccentricity for $X$ group. (f) Observed configuration for $X$ group. 


\section{DISCUSSION}

Some progress has been made in answering the question of how people judge the dissimilarities of pairs of rectangles.

Different people judge them differently: Some peopleour two studies combined suggest it may be roughly $40 \%$-base their ratings on physical differences in width and height of the stimuli. In a $4 \times 4$ stimulus design, they therefore distinguish between all 16 rectangles.

A second group of subjects of roughly the same size bases its judgments on size and eccentricity. These are both linear, not quadratic, functions of physical height and width. Such subjects distinguish only among 10 different rectangles in the above design, because they disregard the rotation that carries flat rectangles into tall rectangles of the same size.

Finally, a smaller group of about $20 \%$ of the subjects bases its judgments mainly on eccentricity, as we defined it in Equation 10b, thus collapsing the design still further. These subjects discriminate effectively only between four equivalence classes. Incidentally, these subjects come closest to interpreting the vague instruction to "rate the dissimilarity" among rectangles in a literal sense, because, technically, "similar" means: of equal shape, disregarding both size and rigid motions.

All subjects, regardless of the strategy they adopt, use a negatively accelerated response function for mapping simple linear combinations of the arguments into the response scale provided by the experimenter, thus arriving at their segmentally subadditive ratings. This response function is well approximated by the tanh function, which, in effect, ensures that all ratings stay below the prescribed upper bound of the response scale.

As a result, the observed dissimilarity ratings will always satisfy the triangle inequality within measurement error (see Note 1). However, we do not believe this finding warrants the inference that the ratings are mediated by some internal spatial representation (a "subjective space") that can be characterized by a particular member of the Minkowski metrics family.

The metric properties of the responses can be explained simply as a consequence of the judgment strategy that subjects use for rating pairs of stimuli. So as not to exceed the upper bound of the rating scale, these strategies necessarily conflict with the basic property of all Minkowski metrics, which is that distances for all collinear triples are additive. Hence, if one were to insist on a metric description of dissimilarity ratings of rectangles, it would have to be some metric for bounded response scales, not any of the Minkowski metrics.

However, once the psychophysical maps between stimuli and ratings are known, not much seems to be gained by invoking spatial models, because then the maps not only permit the prediction of the outcome of the MDS analysis, but also enable us to predict the numerical values of the dissimilarity ratings more parsimoniously than the coordinate vectors of any presumed subjective space.
Birnbaum (1982, 1983) has pointed toward more promising horizons with his emphasis on "scale convergence." Speaking broadly, this means psychophysical research results will be of lasting value only if they can be shown to be invariant over a wide range of tasks, and any changes in parameter values can be explicitly accounted for by changes in the experimental conditions. The goal of establishing such invariances becomes easier once the psychophysical maps have been made explicit, because we then need to monitor only a small number of parameters.

We already know a great deal from classical, 1-D psychophysics about how task, context, and instruction can alter the relationship between physical measures and “subjective scales"' (e.g., Baird, 1970; Birnbaum, 1982; Eisler \& Roskam, 1977; Ekman, 1963; Engen \& Levey, 1955; Garner, 1954; Gigerenzer, 1983; Goude, 1962; Parducci, 1982; Teghtsoonian \& Teghtsoonian, 1971). These findings, while arguing against all-encompassing "psychophysical laws," need not distract us from searching for empirical regularities and invariances, nor should they tempt us to average away systematic variations as "error" in the name of parsimony and generality which, in this case, would simply mask lawful empirical relations: "Instead, it can be argued that any complete theory of psychophysics must give account of the response procedure and contextual effects in order to explain the lawful numerical changes that result as a function of these variables"' (Birnbaum, 1982, p. 407).

The results reported here seem to corroborate Birnbaum's (1982) repeated findings that subjects tend to base their numerical responses on differences, rather than ratios, of "subjective scale values." In effect, we have extended Birnbaum's findings to dissimilarity ratings of 2-D stimuli. A priori, dissimilarity ratings of rectangles might just as well have been based on ratio judgments, because a good deal of previous psychophysical works shows that magnitude estimates of linear extent and area, for example, are often veridical (in the sense that the exponent of the power "law" is unity, e.g., Baird, 1970, p. 50). We found that the difference judgments could be tied directly to the physical dimensions defining the rectangles, and did not have to settle for differences on an underlying subjective scale distinct from the physical scale. Because we were also able to explicate the response function parametrically as an MBR, we are left with very few parameters, perhaps no more than two per strategy group.

If it is possible to explicate such maps, the search for invariances, on one hand, and the pursuit of systematic, experimenter-induced changes, on the other, reduces to two basic problems: (1) monitor quantitative changes in a small number of parameters characterizing the psychophysical maps within each strategy group, and (2) monitor qualitative changes and invariances reflected in strategies characterizing the subgroups. Concretely, one may ask whether subjects can be induced to adopt a particular rating strategy by rephrasing the instruction (e.g., M. Teghtsoonian, 1965), by manipulating the context (e.g., Birn- 
baum, 1982; Parducci, 1982), or by manipulating the presentation sequence (e.g., Ronacher, 1984), and whether such manipulations affect the weights that define the psychophysical maps.

We emphasize that our attempt to account for our data within the framework of the MBR hypothesis was limited to data obtained with bounded rating scales and to physically well-defined stimuli. There is no obvious reason why the specific maps we reported should generalize to entirely different stimulus material-for example, irregular 2-D stimuli such as photographs of human faces-or to different tasks such as magnitude estimation. That is, our results are not meant as "a sharp test of the need for scaling in general" (a reviewer, emphasis his) because they are limited to a specific rating task on specific stimuli.

We attribute the relatively good fit we obtained for each of the psychophysical maps to our prior classification into relatively homogeneous strategy groups. Whether this will be possible depends, among other things, on the nature of the stimuli and the specificity of the judgment task. In the present instance, the stimulus dimensions were relatively well defined (the only two obvious choices seem to be area/shape or height/width) and the task was relatively ill-defined, leaving room for several different simple combination rules to arrive at consistent responses. If these two conditions are not met simultaneously, it may be harder or impossible to find such parametric maps.

\section{REFERENCES}

BAIRD, J. C. (1970). Psychophysical analysis of visual space. Oxford: Pergamon Press.

BiRnBaUm, M. H. (1982). Controversies in psychological measurement. In B. Wegner (Ed.), Social attitudes and psychological measurement. Hillsdale, NJ: Erlbaum.

Birnbaum, M. H. (1983). Scale convergence as a principle for the study of perception. In H. G. Geissler (Ed.), Modern issues in perception. Berlin: VEB.

BoRG, I., \& LEUTNER, D. (1983). Dimensional models for the perception of rectangles. Perception \& Psychophysics, 34, 257-267.

EISLER, H., RosKaM, E. E. (1977). Multidimensional similarity: An experimental and theoretical comparison of vector, distance and set theoretic models. Acta Psychologica, 5, 1-46, 335-363.

EkMAN, G. (1963). A direct method for multidimensional ratio scaling. Psychometrika, 28, 33-41.

ENGEN, T., \& LEVY, N. (1955). The influence of standards on psychophysical judgments. Perceptual \& Motor Skills, 5, 193-197.

GaRner, W. R. (1954). Context effects and the validity of loudness scales. Journal of Experimental Psychology, 48, 218-224.

GIGERENZER, G. (1983). Über die Anwendung der Informationsintegrationstheorie auf entwicklungspsychologische Problemstellungen: Eine Kritik. Zeitschrift fur Entwicklungspsychologie und Padagogische Psychologie, 15, 101-120.

GouDE, G. (1962). On fundamental measurement in psychology. Stockholm: Almquist \& Wiksell.

KrANTZ, D., \& TVERSKY, A. (1975). Similarity of rectangles: An analysis of subjective dimensions. Journal of Mathematical Psychology, 12, 4-34.

Noma, E., \& Johnson, J. (1977). Constraining nonmetric multidimensional scaling configurations (Tech. Rep. No. 60). Ann Arbor, MI: University of Michigan, Human Performance Center.

ParducC, A. (1982). Category ratings: Still more contextual effects. In B. Wegner (Ed.), Social attitudes and psychophysical measurement. Hillsdale, NJ: Erlbaum.
RONACHER, B. (1984). Human pattern recognition: Evidence for switching between strategies in analyzing complex stimuli. Biological Cybernetics, 51, 205-210.

Rosch, E. (1977). Human categorization. In N. Warren (Ed.), Advances in cross cultural psychology (Vol. 1). London: Academic Press.

SchönemanN, P. H. (1982). A metric for bounded response scales. Bulletin of the Psychonomic Society, 15, 317-319.

SchönemanN, P. H. (1983). Some theory and results for metrics for bounded response scales. Journal of Mathematical Psychology, 27, 311-324.

SCHÖNEMANN, P. H. (1985). On the formal differentiation of traces and determinants. Multivariate Behavioral Research, 20, 113-139.

Schönemann, P. H., \& BoRG, I. (1981). On the interaction between area and shape. In I. Borg (Ed.), Multidimensional data representations: When and why. Ann Arbor, MI: Mathesis.

Schönemann, P. H., DoRCey, T., KienapPle, K. (1985). Subadditive concatenation in dissimilarity judgments. Perception \& Psychophysics, 38, 1-17.

SChönemanN, P. H. \& KienapPle, K. (1984). Artefakte in der mehrdimensionalen Skalienung. Zeitschrift für Experimentelle und $A b$ gewandte Psychologie, 31, 483-506.

Schulz, U., \& MAY, T. (1985). Die Erhebung sozialer Orientierungen mit Rangordnungs- und Paarvergleichsverfahren (Bericht Nr. 6). Universität Bielefeld. (English version submitted to European Journal of Social Psychology)

SIXTL, F., \& WENDER, K. (1964). Der Zusammenhang zwischen multidimensionalem Skalieren und Faktorenanalyse. Biometrische Zeitschrift, 6, 251-261.

Teghtsoonian, M. (1965). The judgment of size. American Joumal of Psychology, 78, 392-402.

Teghtsoonian, M., \& Teghtsoonian, R. (1971). How repeatable are Stevens' power law exponents for individual subjects? Perception \& Psychophysics, 10, 147-149.

WENDER, K. (1969). Die psychologische Interpretation nichteuklidischer Metriken in der multidimensionalen Skalierung. Unpublished doctoral dissertation, Technische Hochschule Darmstadt.

WENDER, K. (1971). A test of independence of dimensions in multidimensional scaling. Perception \& Psychophysics, 10, 30-32.

WIENER-EHRLICH, W. K. (1978). Dimensional and metric structures in multidimensional stimuli. Perception \& Psychophysics, 24, 399-414.

\section{NOTES}

1. A reviewer asked about the MDS algorithm and the problem of asymmetric dissimilarity ratings.

MIS algorithm. In contrast to the analyses presented in Schönemann et al. (1985), which were run on a mainframe computer with MRSCAL, a scaling program kindly provided to us by E. Roskam (see Eisler \& Roskam, 1977, for details), we wrote our own program for the MDS analyses reported here to be able to analyze the data on an IBM PC. This program uses a gradient-method algorithm with externally controllable step size. The gradients are based on the derivatives of the cityblock metric (Equation 2) and the $\operatorname{MBR}(k)$,

$$
\mathrm{d}^{*}=\left(a^{*}+b^{*}\right) /\left(1+a^{*} b^{*} / k^{2}\right)
$$

where $k$ is the effective upper bound (we used $k=L=9$ ) and $d^{*}$ is the observed dissimilarity. This approach dispenses with the need to invoke the inverse hyperbolic tangent transformation to fit the MBR. Convergence, which was rather slow, was measured by the length of the gradients.

Asymmetry. Although we distinguished between $d_{i j}$ and $d_{j i}$ at the within-subject level, we did not expect or find any systematic departures from symmetry in the present data or the previously reported $9 \times 9$ data, inasmuch as the stimulus pairs $(x, y)$ differed from $(y, x)$ solely by a position reversal on the screen. We therefore based the MDS analyses on symmetrized averages in each group.

However, as noted in the text, the MDS analyses do suppress another type of asymmetry, the "saliency effect" of the dimension with the larger difference which, once taken into account, clearly improves the fit of 
the one-parameter maps. This is one more reason why we prefer the psychophysical maps, which easily accommodate this type of asymmetry, over MDS solutions based on distance concepts that do not. Put differently, we consider the dynamic framework of concept formation (e.g., Rosch, 1977) to be more fruitful than the static framework of a geometric representation for explaining data of this type.

2. Schulz and May (1985, p. 2) reported that the same argument (as Equation 18) also describes payoff preferences in game theory. The connection, if any, is not clear to us at this time.

\section{APPENDLX \\ The $M$ Dimensional Generalization}

Let $I:=[0,1) \in \operatorname{Re}$ be the half-open interval from 0 to 1 . On defining the binary operation $\oplus$ in $I$ :

$$
\begin{aligned}
\oplus: a \oplus b:=(a+b) /( & 1+a b) \\
& =\tanh (u+v) \leq u+v \text { (subadditivity) }
\end{aligned}
$$

(where $a=\tanh (u), b=\tanh (v), a, b \in I$ ), one finds that $\oplus$ is monotone, commutative, and associative, that 0 is the neutral element, and that the inverse operation $\theta$, implicitly defined by

$$
\ominus:=(a \oplus b) \ominus b=a, \text { for all } a, b \in I,
$$

is

$$
\begin{aligned}
a \in b=(a-b) /(1-a b) & \\
& =\tanh (u-v) \geq u-v \text { (suprasubtractivity). }
\end{aligned}
$$

Now let $x, y \in I$ with $x<y$. Then $x \oplus(y \ominus x)=y$, by the above definitions of $\oplus$ and $\theta$, that is, $y \theta x$ is the length of the line segment from $x$ to $y$, which under $\oplus$ has to be added to $x$ to give $y$.

Hence, the MBR (Equation 3) becomes, in terms of coordinates:

$$
d_{i j}=\tanh \left(\tanh ^{-1}\left|x_{i 1}-x_{j 1}\right|+\tanh ^{-1}\left|x_{i 2}-x_{j 2}\right|\right),
$$

because, for example, $u=\tanh ^{-1}(a)=\tanh ^{-1}\left(\left|x_{j 1}-x_{i 1}\right|\right)$.

More generally, on defining the $m$-fold subadditive sum,

$$
\oplus_{k} z_{k}:=z_{l} \oplus \ldots \oplus z_{m} \text { in } I^{m},
$$

one obtains as $m$-dimensional MBR in $I^{m}$ :

$$
d_{i j}:=\tanh \left[\Sigma_{k} \tanh ^{-1}\left(\left|x_{i k}-x_{j k}\right|\right)\right], \quad k=l, m,
$$

which, for $m=1$, gives $d_{i j}=\tanh \left(u_{i}-u_{s}\right)$, which we used in Equation 7 to find the psychophysical maps for squares.

(Manuscript received August 8, 1986; revision accepted for publication April 7, 1987.)

\section{Notice}

\section{Nominations for the Editorship of Memory \& Cognition}

Nominations are solicited for the editorship of Memory \& Cognition. The term of the present editor, Alice F. Healy, expires at the end of 1989 . The new editor will begin to receive manuscripts in 1989, with an official 4-year term to begin January 1,1990 . Nominations must be submitted by March 1, 1988.

Nominations (including self-nominations) should be sent to:

Richard M. Shiffrin Chair, Memory \& Cognition Search Committee Department of Psychology

Indiana University

Bloomington, IN 47405 